\title{
Coronary CT Angiography with Knowledge-Based Iterative Model Reconstruction for Assessing Coronary Arteries and Non-Calcified Predominant Plaques
}

\author{
Tao Li, MD, Tian Tang, BA, Li Yang, MD, Xinghua Zhang, MD, Xueping Li, BS, Chuncai Luo, BS \\ All authors: Department of Radiology, Chinese People's Liberation Army General Hospital, Beijing, China
}

Objective: To assess the effects of iterative model reconstruction (IMR) on image quality for demonstrating non-calcific highrisk plaque characteristics of coronary arteries.

Materials and Methods: This study included 66 patients (53 men and 13 women; aged 39-76 years; mean age, $55 \pm 13$ years) having single-vessel disease with predominantly non-calcified plaques evaluated using prospective electrocardiogramgated 256-slice CT angiography. Paired image sets were created using two types of reconstruction: hybrid iterative reconstruction (HIR) and IMR. Plaque characteristics were compared using the two algorithms. The signal-to-noise ratio (SNR) and contrastto-noise ratio (CNR) of the images and the CNR between the plaque and adjacent adipose tissue were also compared between the two reformatted methods.

Results: Seventy-seven predominantly non-calcified plaques were detected. Forty plaques showed napkin-ring sign with the IMR reformatted method, while nineteen plaques demonstrated napkin-ring sign with HIR. There was no statistically significant difference in the presentation of positive remodeling, low attenuation plaque, and spotty calcification between the HIR and IMR reconstructed methods (all $p>0.5$ ); however, there was a statistically significant difference in the ability to discern the napkin-ring sign between the two algorithms $\left(\chi^{2}=12.12, p<0.001\right)$. The image noise of IMR was lower than that of HIR (10 $\pm 2 \mathrm{HU}$ versus $12 \pm 2 \mathrm{HU} ; p<0.01$ ), and the SNR and CNR of the images and the CNR between plaques and surrounding adipose tissues on IMR were better than those on HIR $(p<0.01)$.

Conclusion: IMR can significantly improve image quality compared with HIR for the demonstration of coronary artery and atherosclerotic plaques using a 256-slice CT.

Keywords: Coronary artery disease; Atherosclerosis; Image reconstruction; Multidetector computed tomography; Computed tomography angiography

\section{INTRODUCTION}

Image reconstruction algorithms play a critical role in

Received July 7, 2018; accepted after revision January 21, 2019.

This study was supported by state key and research and development project grant 2016YFC1300300 and NSFC grant 81371547.

Corresponding author: Li Yang, MD, Department of Radiology, Chinese People's Liberation Army General Hospital, Number 28, Fu Xing Road, Hai Dian District, Beijing 100853, China.

- Tel: (8610) 66939564 - Fax: (8610) 66939564

-E-mail:Yangli301@yeah.net

This is an 0pen Access article distributed under the terms of the Creative Commons Attribution Non-Commercial License (https://creativecommons.org/licenses/by-nc/4.0) which permits unrestricted non-commercial use, distribution, and reproduction in any medium, provided the original work is properly cited. coronary computed tomographic angiography (CCTA). The conventional filtered back projection (FBP) algorithm results in a substantial increase in image noise and streak artefacts with a reduction in the tube voltage and output, leading to impaired diagnostic image quality $(1,2)$.

Iterative reconstruction (IR) algorithms are advantageous in reducing the noise associated with FBP reconstruction algorithms (3-5). The hybrid IR (HIR) algorithm facilitates noise and radiation dose reduction compared with FBP (4, 6-8). Iterative model reconstruction (IMR), a knowledgebased fully IR algorithm, is the latest advancement in the field of reconstruction technology, and has been applied to CCTA (9-12). Previous studies on IMR mainly focused on the quality of coronary artery images and radiation dose reduction $(13,14)$. However, it has not been known whether 
image quality for the identification of vulnerable coronary atherosclerotic plaques could be improved using IMR.

The detection of coronary atherosclerotic plaque and identification of plaque vulnerability are important in devising appropriate intervention strategies for optimal prognosis. Studies showed that most acute coronary syndromes result from the rupture of vulnerable plaques and subsequent thrombus formation rather than from the severity of luminal stenosis (15). However, the demonstration of non-calcified components of coronary artery plaques using the HIR algorithm is often difficult. We hypothesized that the IMR algorithm is better than the HIR in the characterization of coronary plaques and detection of vulnerable plaques. The purpose of this study was to assess the image quality of IMR in demonstrating non-calcified or vulnerable components of coronary artery plaques in comparison with that of HIR.

\section{MATERIALS AND METHODS}

\section{Patient Selection}

This research protocol was approved by the Institutional Review Board, and informed consent was obtained from all patients before examination in this prospective study. This study enrolled patients with suspected coronary artery diseases who had undergone CCTA from August 2016 to January 2017, in our institution. Data were collected from subjects having single-vessel disease with noncalcified coronary plaques and significant stenosis $(\geq 50 \%$ in diameter) on CCTA. Exclusion criteria for CCTA included subjects with renal dysfunction (glomerular filtration rate $<60 \mathrm{~mL} / \mathrm{min}$ ), arrhythmia, known allergy to iodinated contrast media, and the inability to sustain a 10-second breath-hold. Finally, 66 subjects (53 men and 13 women; aged $39-76$ years; mean age, $55 \pm 13$ years) were included.

\section{CCTA Protocol}

CCTA was performed on a 256-slice CT scanner (Brilliance iCT; Philips Healthcare, Cleveland, $\mathrm{OH}$, USA). In all patients with a heart rate $(H R)>65$ beats per minute (bpm), a $\beta$-blocker (metoprolol tartrate; AstraZeneca, London, UK) was orally administered at the dose of $25-50 \mathrm{mg}$ to achieve the target $\mathrm{HR} \leq 65 \mathrm{bpm}, 2$ hours before CT examinations. Administration of sublingual nitroglycerin (LGC, Teddington, UK) for coronary vasodilation was performed at the time of imaging. CCTA was performed using a prospective electrocardiogram (ECG) triggering protocol using the following parameters: slice configuration, $128 \times 0.625 \mathrm{~mm}$; gantry rotation time, $270 \mathrm{~ms}$; pitch, 0.18 ; tube voltage, 100 $\mathrm{kVp}$; effective tube current time product, $600 \mathrm{mAs}$ with ECGdependent tube current modulation; and slice thickness, $0.8 \mathrm{~mm}$ with slice increment of $0.45 \mathrm{~mm}$. A bolus-tracking technique was used to monitor the CT value of the ascending aorta with a trigger threshold of $120 \mathrm{HU}$. The scans were started 5 seconds after the trigger. Each patient received $70 \mathrm{~mL}$ of contrast media (iopromide, Ultravist ${ }^{\circledR}$ 370; Bayer Healthcare, Berlin, Germany) intravenously at the speed of $5 \mathrm{~mL} / \mathrm{s}$, followed by $40 \mathrm{~mL}$ of saline at the same injection speed.

\section{Coronary CT Image Post-Processing and Data Analysis}

The $\mathrm{CT}$ data were respectively reconstructed with an HIR algorithm (iDose4-level; Philips Healthcare) and an implementation of the new knowledge-based IR algorithm (IMR-level 1) for each subject. The reconstructed parameters were the same, including $0.8 \mathrm{~mm}$ thickness with $0.45 \mathrm{~mm}$ increment, $512 \times 512$ in matrix, $250 \mathrm{~mm}$ field of view and a standard cardiac reconstruction kernel (XCB). All CCTA data were transferred to a computer workstation (Extended Brilliance WorkspaceV4.5.2; Philips Healthcare).

Maximum intensity projection (MIP), multi-planar reconstruction (MPR), and curved-planar reconstruction images were reconstructed and used for detecting coronary plaque by a radiologist with 5 years of experience in CCTA. An MIP image of the long axis of the coronary artery was reconstructed to identify lesions with significant stenosis, and then cross-sectional MPR images of $1 \mathrm{~mm}$ thickness through the plaque were reconstructed for demonstrating the plaque and measuring the contrast-to-noise ratio (CNR) between the plaque and adjacent adipose tissue. The CT value of the aortic root was measured on axial source image with both HIR and IMR algorithms. The region of interest (ROI) in the aortic root was about $200 \mathrm{~mm}^{2}$. The CT values were also measured in the lumens of the left main (LM) trunk, proximal, middle, and distal segments of the right coronary artery (RCA), left anterior descending (LAD) artery, and proximal and distal segments of the left circumflex (LCX) artery. The round ROI was placed in the center of the vessels and was neither small enough to be affected by pixel variability nor large enough to approach the edge of the vessels (Figs. 1, 2). The CT values of plaques were measured on both HIR and IMR-reformatted axial images using ROIs with the same size and location. 

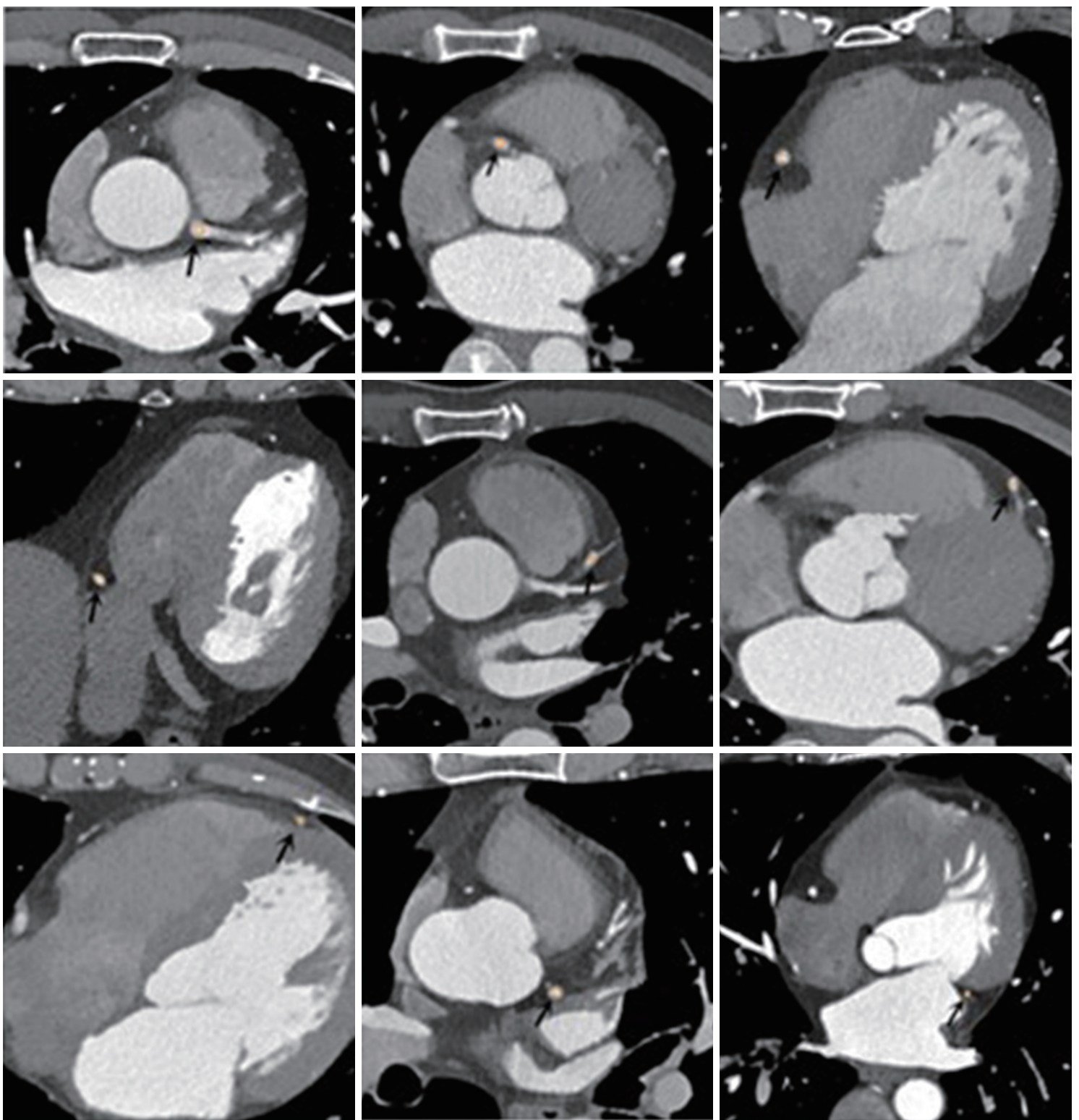

Fig. 1. CT value measurement in coronary artery segments (arrows) on IMR images. CT values were measured in lumen of left main trunk, proximal, middle, and distal segments of right coronary artery, LAD artery, and proximal and distal segments of left circumflex artery. IMR $=$ iterative model reconstruction, $\mathrm{LAD}=$ left anterior descending

Each ROI was located at the lowest attenuation area of the plaque and drawn as large as possible. Microcalcifications or other disturbing structures were carefully avoided. The $\mathrm{CT}$ values of the adjacent perivascular adipose tissues were also measured (Fig. 3). Noise was defined as the standard deviation (SD) measured in the background (air), anterior to the patients' chest wall. The area of the ROI for measuring noise was about $50 \mathrm{~mm}^{2}$ (Fig. 4). The measurement in each ROI was performed three times, and the average value was calculated. The signal-to-noise ratio (SNR) was calculated for intravascular attenuation and plaque attenuation. CNR was calculated as follows: ([attenuation of vessel lumen or attenuation of plaque] - [attenuation of the adjacent perivascular adipose tissue]) / image noise. The coronary segmentation was determined according to the Society of Cardiovascular CT guidelines for the interpretation and reporting of CCTA (16).

High-risk plaque analysis was performed using the follow criteria: low-attenuation plaque (plaque attenuation lower than $60 \mathrm{HU}$ ), napkin-ring sign, spotty calcifications (calcification $<3 \mathrm{~mm}$ in size), and positive remodeling. Napkin-ring sign was defined as an outer high-attenuation rim (<200 HU) with an inner hypo-attenuation area (< 130 $\mathrm{HU})$, which was not adjacent to a calcification and present 

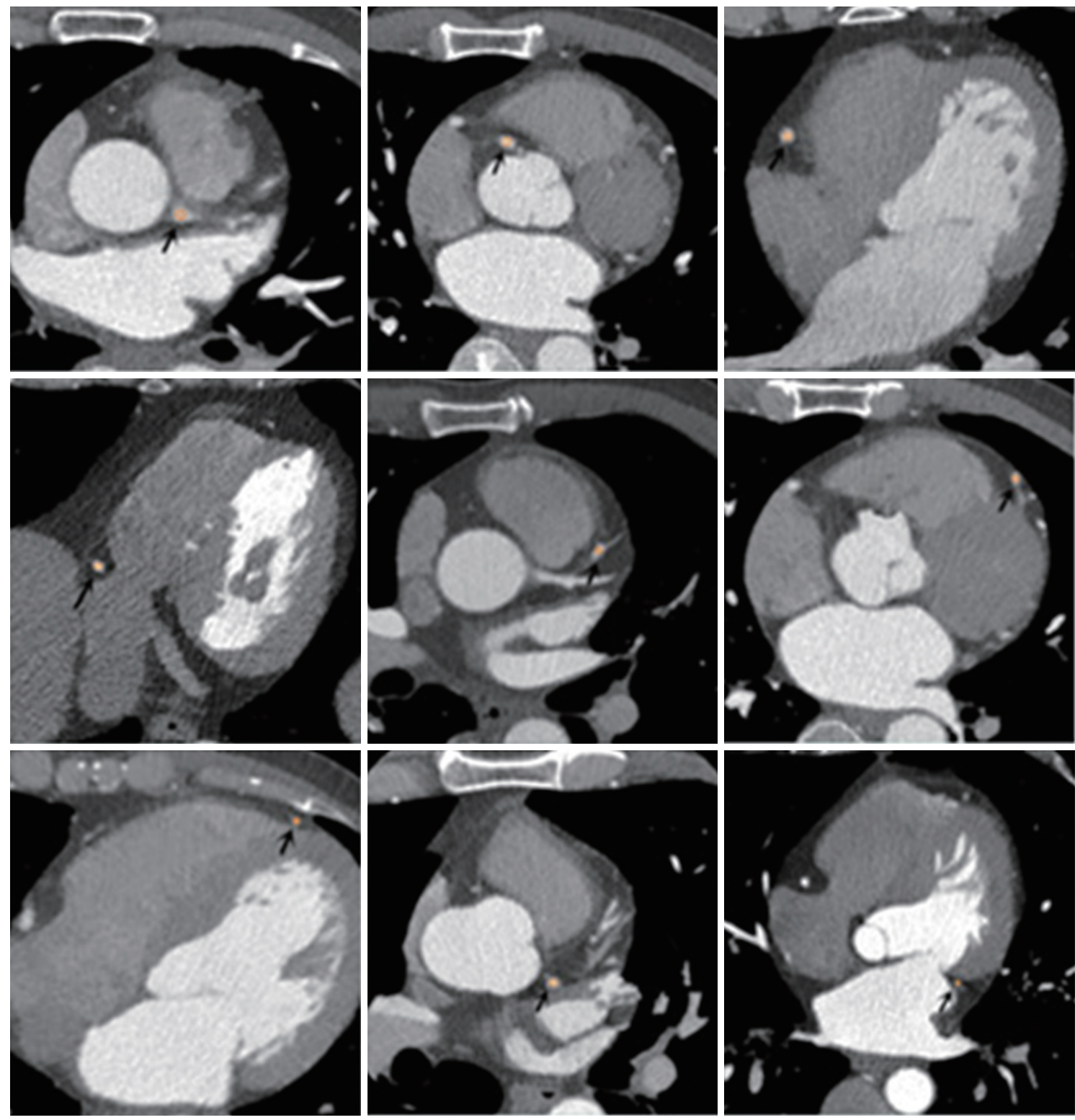

Fig. 2. CT value measurement in coronary artery segments (arrows) on HIR images in same patient. HIR = hybrid iterative reconstruction

on a minimum of two adjacent $1 \mathrm{~mm}$ axial slices. We carefully checked the non-contrast CT for microcalcifications when we evaluated a napkin-ring sign. The remodeling index was calculated as the ratio of the maximal crosssectional vessel diameter, including the plaque and lumen, and its closest proximal normal reference vessel diameter (17). A remodeling index $>1.05$ was considered as positive remodeling (18).

Two experienced radiologists with 10 and 5 years of experience with CCTA, respectively, blinded to image algorithms, identified plaque characteristics independently and collectively resolved any differences in their readings.

\section{Statistical Analysis}

Continuous variables were presented as mean $\pm S D$, and categorical variables were expressed as frequencies or percentages. A paired $t$ test was used to determine the differences in image noise, vascular attenuation, plaque attenuation, adipose tissue attenuation surrounding plaque, SNR, and CNR between the two image reconstruction methods. Chi-square test was performed to compare the difference between the HIR and IMR algorithms in detecting plaque characteristics. Cohen's kappa test was used to analyze the inter-observer agreement in discerning the plaque characteristics. The $\kappa$ values were defined as 

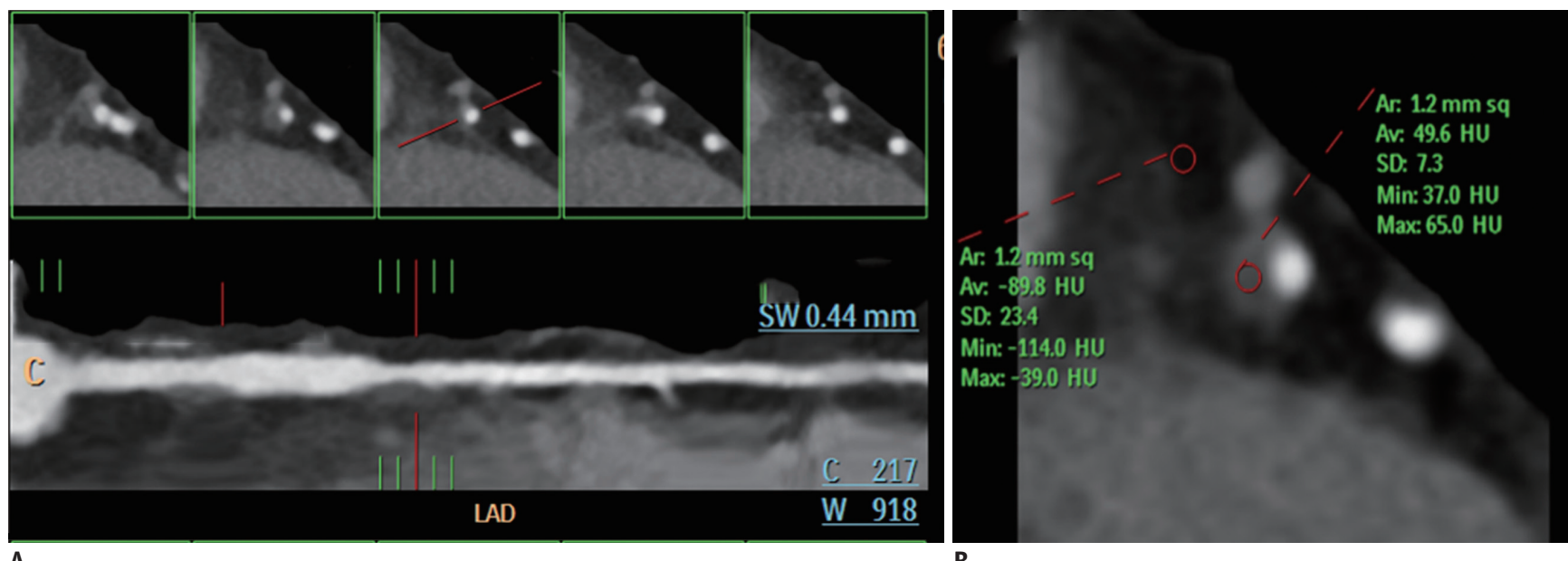

Fig. 3. Plaque identification and CT value measurement.

A. Non-calcified plaque was detected on proximal segment of LAD on curved reformation. B. CT value of plaque and adjacent perivascular adipose tissue measurement. $\mathrm{Ar}=$ area, $\mathrm{Av}=$ average, $\mathrm{Max}=$ maximum, $\mathrm{Min}=$ minimum, $\mathrm{SD}=$ standard deviation

follows: $0-0.2$, poor agreement; $0.21-0.40$, fair agreement; $0.41-0.60$, moderate agreement; $0.61-0.80$, substantial agreement; and 0.81-1.00, almost perfect agreement. A two-tailed $p$ value $<0.05$ was considered statistically significant. All statistical analyses were conducted by SPSS 18.0 for Windows (SPSS Inc., Chicago, IL, USA).

\section{RESULTS}

\section{Coronary Atherosclerotic Plaque Characteristics}

Seventy-seven non-calcified or predominantly noncalcified plaques with significant coronary stenosis were detected. Two plaques were located in $L M, 51$ in LAD, 23 in RCA, and 1 in LCX.

A total of $72(93.5 \%)$ high-risk plaques were detected on IMR images, including $42(54.5 \%)$ showing low attenuation, $40(51.9 \%)$ with napkin-ring sign (Figs. 5, 6), 18 (23.4\%) with spotty calcifications, and $59(76.6 \%)$ with positive remodeling. There were 70 (90.9\%) high-risk plaques identified on HIR images, including $41(53.2 \%)$ showing low attenuation, $19(24.7 \%)$ with napkin-ring sign, $18(23.4 \%)$ with spotty calcifications, and $57(74.0 \%)$ with positive remodeling. The chi-square test showed no statistically significant difference in the presentation of low attenuation plaque, spotty calcification, or positive remodeling between the HIR and IMR reconstruction methods, but there was a statistically significant difference in the discernment of the napkin-ring sign between the two algorithms $\left(\chi^{2}=12.12, p\right.$ $<0.001$ ) (Table 1).

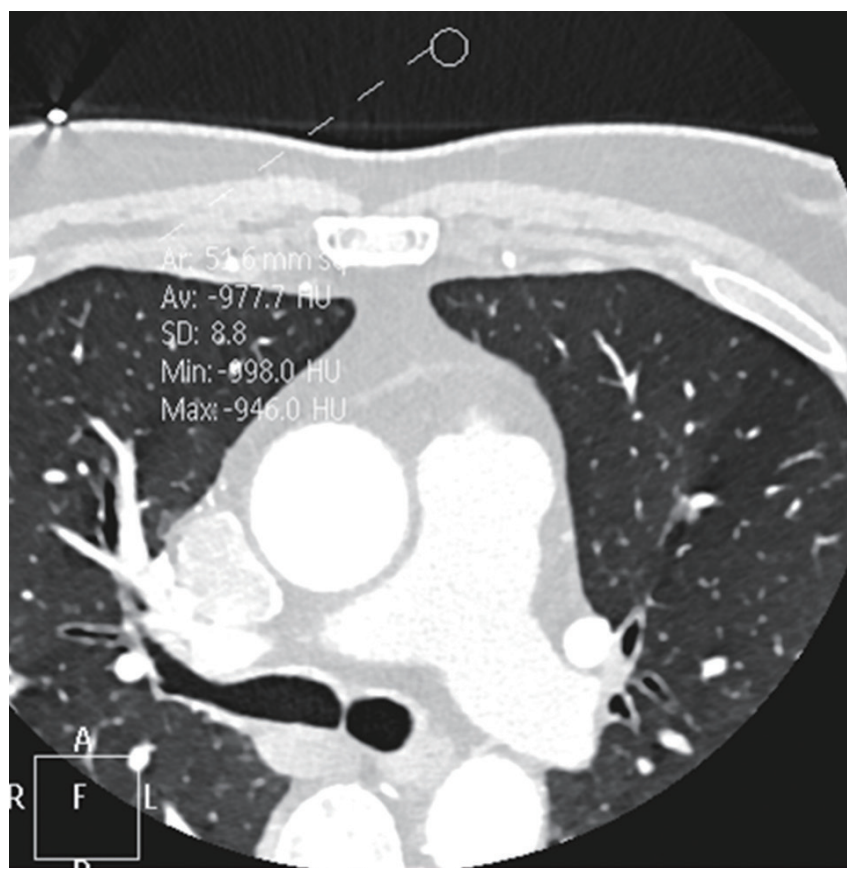

Fig. 4. Noise measurement. Noise was defined as SD measured in background (air) anterior to patients' chest wall. Area of region of interest for measuring noise was about $50 \mathrm{~mm}^{2}$.

\section{Quantitative Analysis}

Table 2 summarizes the results of image quality between the HIR and IMR reconstruction algorithms. The noise with IMR was significantly lower than that with $\operatorname{HIR}(p<$ 0.001). The attenuation of the aortic root with IMR was not different from that with the HIR reconstruction method $(p=0.674)$. The CT values of the coronary artery segments measured using IMR were significantly greater compared to those using HIR $(p<0.001)$. The CT values of adipose 


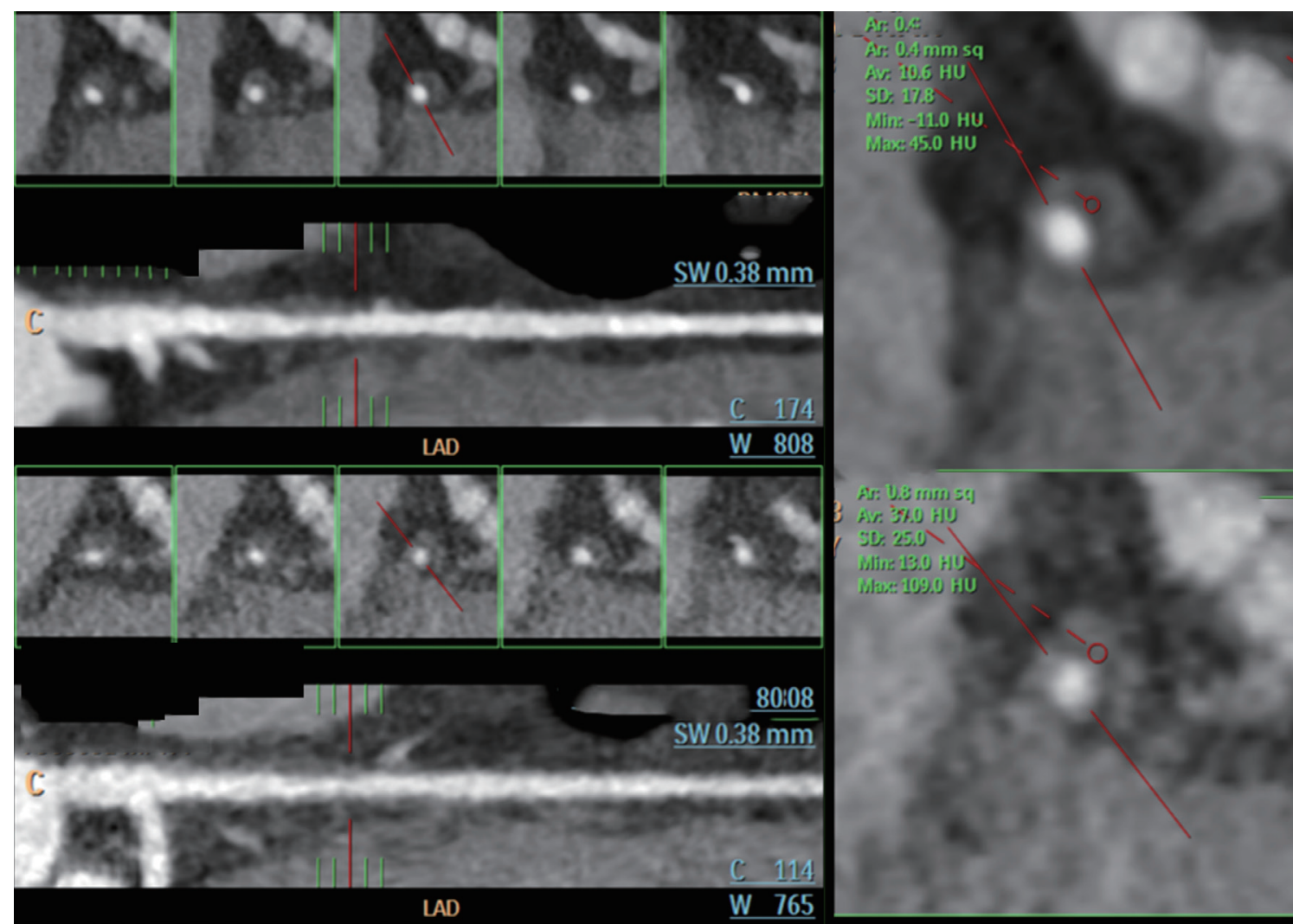

Fig. 5. Representative figures of napkin-ring sign in 47-year-old male patient. Top figures show that napkin-ring sign of LAD plaque can be detected using IMR algorithm, but not on HIR image (bottom figures).

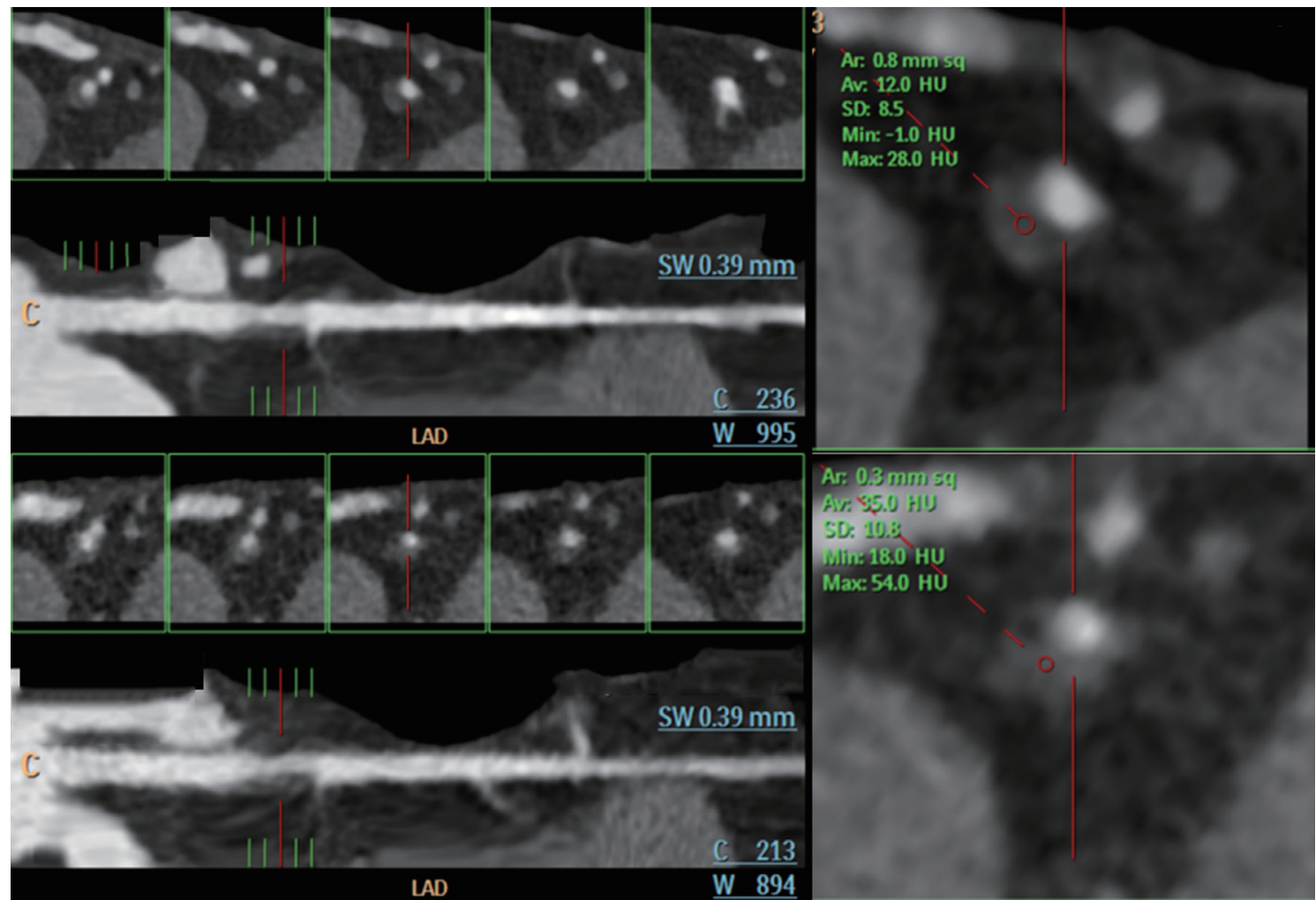

Fig. 6. Representative figures of napkin-ring sign in 64-year-old female patient. Top figures show that napkin-ring sign of LAD plaque can be detected using IMR algorithm, but not on HIR image (bottom figures). 
tissues surrounding the vessels measured using IMR were significantly lower compared to those using $\operatorname{HIR}(p<0.05)$, except for the LAD middle segment $(p=0.954)$ and $L C X$ distal segment $(p=0.100)$. The SNR and CNR using IMR were significantly greater compared to those using the HIRreformatted algorithm $(p<0.001)$ (Table 3$)$. Table 4 presents the quantitative analysis of coronary atherosclerotic plaques between the two algorithms. The CT value of the plaques was significantly higher with IMR than that with $\operatorname{HIR}(p<$ 0.05 ), and the CT value of the surrounding adipose tissue

Table 1. Plaque Characteristics Demonstration between HIR and IMR Algorithms

\begin{tabular}{|c|c|c|c|c|}
\hline Characteristics & $\operatorname{HIR}(n=77)$ & $\operatorname{IMR}(n=77)$ & $\chi^{2}$ & $P$ \\
\hline No. of high-risk plaques & $70(90.9 \%)$ & $72(93.5 \%)$ & 0.36 & 0.548 \\
\hline Positive remodeling & $57(74.0 \%)$ & $59(76.6 \%)$ & 0.14 & 0.709 \\
\hline Low attenuation & $41(53.2 \%)$ & $42(54.5 \%)$ & 0.03 & 0.872 \\
\hline Spotty calcifications & $18(23.4 \%)$ & $18(23.4 \%)$ & 0.00 & 1.000 \\
\hline Napkin-ring sign & $19(24.7 \%)$ & $40(51.9 \%)$ & 12.12 & $<0.001$ \\
\hline
\end{tabular}

was significantly lower with IMR than that with $\operatorname{HIR}(p<$ 0.05). Compared with HIR, the SNR and CNR between plaque and surrounding adipose tissue were significantly improved with IMR $(p<0.001)$. Cohen's kappa test showed that the agreement between the two observers for discerning plaque characteristics was 0.77 (Kappa $=6.73, p<0.001)$.

\section{DISCUSSION}

In the present study, we evaluated coronary atherosclerotic plaques with knowledge-based IMR in CCTA. The major findings of the study were twofold: first, IMR demonstrates the napkin-ring sign of plaque better than $\mathrm{HIR}$; second, IMR can significantly reduce the image noise and improve the image quality and CNR between coronary atherosclerotic plaques and surrounding adipose tissue compared with HIR on 256-slice CCTA.

HIR can decrease image noise and artefacts to some degrees depending on the proportion of iterative blending with FBP. However, a certain amount of image noise and

Table 2. Image Noise, СT Values of Aortic Root, Coronary Artery Segments and Adipose Tissue of Each Region between HIR and IMR Algorithms

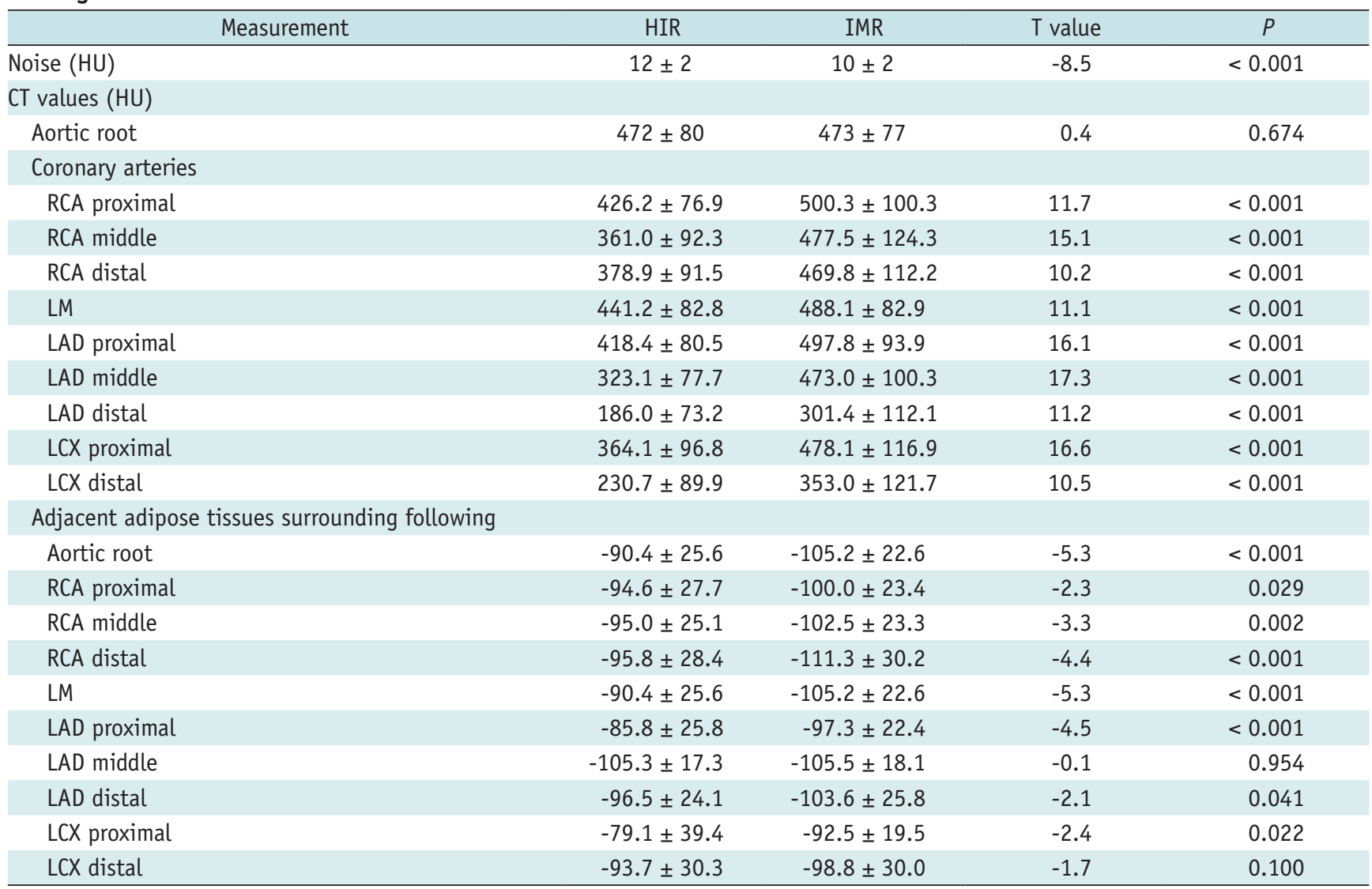

$\mathrm{LAD}=$ left anterior descending, $\mathrm{LCX}=$ left circumflex, $\mathrm{LM}=$ left main, $\mathrm{RCA}=$ right coronary artery 
artefacts remain in a low-dose scanning protocol that uses an HIR (19).

IMR is an advanced, knowledge-based IR algorithm that controls image noise using data statistics, image statistics, and system modeling (20). There are two IMR cardiac settings (Cardiac Routine and Cardiac Sharp; Philips Healthcare), each with three levels (L1, L2, and L3). In our hospital, we targeted the lowest noise reduction, Level 1, with the cardiac routine setting for visualizing coronary arteries and soft tissue. IMR does not involve blending with FBP images and provides full IR. Originally, model-based IR

Table 3. SNR of Images and CNR between Vessels and Adjacent Epicardial Adipose Tissues between HIR and IMR Algorithms

\begin{tabular}{lccrc}
\hline Measurement & HIR & IMR & T value & $P$ \\
\hline SNR & & & & \\
\hline Aortic root & $38 \pm 10$ & $48 \pm 14$ & 6.7 & $<0.001$ \\
\hline RCA proximal & $35.1 \pm 8.5$ & $51.8 \pm 13.7$ & 10.0 & $<0.001$ \\
\hline RCA middle & $29.9 \pm 9.6$ & $49.9 \pm 17.2$ & 10.6 & $<0.001$ \\
\hline RCA distal & $31.2 \pm 8.7$ & $49.1 \pm 15.6$ & 9.4 & $<0.001$ \\
\hline LM & $36.2 \pm 8.5$ & $50.7 \pm 12.7$ & 8.9 & $<0.001$ \\
\hline LAD proximal & $34.3 \pm 8.3$ & $51.5 \pm 12.8$ & 10.4 & $<0.001$ \\
\hline LAD middle & $26.4 \pm 7.2$ & $49.2 \pm 14.2$ & 13.5 & $<0.001$ \\
\hline LAD distal & $15.6 \pm 7.5$ & $30.7 \pm 11.8$ & 11.8 & $<0.001$ \\
\hline LCX proximal & $29.8 \pm 8.8$ & $49.9 \pm 16.3$ & 10.9 & $<0.001$ \\
\hline LCX distal & $27.0 \pm 10.7$ & $36.8 \pm 14.8$ & 11.0 & $<0.001$ \\
\hline CNR & & & & \\
\hline Aortic root & $46.6 \pm 11.1$ & $60.0 \pm 13.4$ & 9.2 & $<0.001$ \\
\hline RCA proximal & $42.9 \pm 9.8$ & $62.2 \pm 15.2$ & 9.9 & $<0.001$ \\
\hline RCA middle & $37.6 \pm 10.2$ & $60.6 \pm 18.3$ & 10.9 & $<0.001$ \\
\hline RCA distal & $39.0 \pm 9.3$ & $60.7 \pm 18.1$ & 9.8 & $<0.001$ \\
\hline LM & $43.7 \pm 9.5$ & $61.7 \pm 14.6$ & 9.1 & $<0.001$ \\
\hline LAD proximal & $41.4 \pm 9.0$ & $61.6 \pm 13.5$ & 10.5 & $<0.001$ \\
\hline LAD middle & $35.1 \pm 7.8$ & $60.3 \pm 16.1$ & 12.5 & $<0.001$ \\
\hline LAD distal & $23.5 \pm 8.2$ & $41.4 \pm 12.1$ & 12.7 & $<0.001$ \\
\hline LCX proximal & $36.2 \pm 9.3$ & $59.5 \pm 17.6$ & 11.0 & $<0.001$ \\
\hline LCX distal & $27.0 \pm 10.7$ & $47.1 \pm 17.3$ & 11.2 & $<0.001$ \\
\hline
\end{tabular}

CNR: (luminal CT values minus CT values of surrounding adipose tissue) divided by noise, SNR: luminal CT values divided by noise. CNR = contrast-to-noise ratio, SNR $=$ signal-to-noise ratio required extended reconstruction time because of its more complicated algorithm than HIR, which restricted its clinical feasibility (12). More recently, the powerful computational ability of IMR has reduced the reconstruction time to within approximately 5 minutes, which has led to routine clinical application of IMR in CCTA (12).

Only a few published clinical studies have demonstrated that the use of a low tube voltage protocol combined with IMR could facilitate dose reduction without compromising image quality in CCTA $(13,14,21,22)$. Our study also showed that IMR could reduce image noise and improve image quality compared with HIR. This is consistent with previous studies. Some studies $(19,23)$ found no difference in CT values in great vessels between IMR and HIR. However, few studies paid attention to the difference in $\mathrm{CT}$ values in small vessels between the two algorithms. Furthermore, whether coronary plaque characteristics could be demonstrated more clearly with IMR is still unknown. To our knowledge, the present study is the first to assess in vivo non-calcified or predominantly non-calcified coronary plaques using the IMR algorithm. Our study revealed that the CT value in the coronary artery was significantly increased with IMR compared with HIR. Our study also found that IMR could strengthen the contrast between plaque and adjacent adipose tissue, which is possibly attributable to the increase in the CT values of plaques and the decrease in the CT value of adjacent adipose tissue with IMR. The increased contrast between plaques and surrounding adipose tissue improves visual evaluation of coronary non-calcified or predominantly non-calcified plaques.

High-risk plaques distinguish the patients who are at a risk for future major adverse cardiac events (MACEs). The high-risk plaque criteria by CCTA include low attenuation plaque, spotty calcification, positive remodeling, and napkin-ring sign. A previous study found that the napkinring sign is the most powerful predictor for MACE (17). Our study shows that a significantly higher proportion of

Table 4. Quantitative Analysis of Coronary Atherosclerotic Plaques between HIR and IMR Algorithms

\begin{tabular}{lcccr}
\hline \multicolumn{1}{c}{ Measurement } & HIR & IMR & T value & \multicolumn{1}{c}{$P$} \\
\hline CT values of plaque (HU) & $51 \pm 34$ & $58 \pm 34$ & 2.5 & 0.013 \\
CT values of surrounding adipose tissue (HU) & $-95 \pm 21$ & $-100 \pm 20$ & -2.1 & 0.037 \\
Noise (HU) & $12 \pm 2$ & $10 \pm 2$ & -8.5 & $<0.001$ \\
SNR & $3.8 \pm 2.2$ & $5.5 \pm 3.1$ & 5.3 & $<0.001$ \\
CNR & $12.0 \pm 3.6$ & $16.0 \pm 4.6$ & 9.4 & $<0.001$ \\
\hline
\end{tabular}

CNR: CT values of plaque minus CT values of adjacent epicardial adipose tissue divided by noise, SNR: CT values of plaque divided by noise. 
napkin-ring sign is visualized on IMR images than on HIR. Therefore, IMR is more advantageous for detecting high-risk plaques than is HIR.

There are a few limitations of this study. First, FBP was not compared with HIR and IMR, although some studies in the literature have reported that HIR can reduce image noise and help obtain images of good quality during CCTA compared with $\operatorname{FBP}(19,24,25)$. Second, diagnostic accuracy in the detection of high-risk plaques was not verified using the gold standard. Because of these limitations, a follow-up study is ongoing in the same patients in our institution.

In conclusion, the knowledge-based IMR algorithm significantly reduces image noise and produces better quality images during CCTA in comparison with HIR. Plaque characteristics can be more clearly discerned with IMR compared with HIR, thereby indicating that this method may enable detection of plaque vulnerability.

\section{Conflicts of Interest}

The authors have no potential conflicts of interest to disclose.

\author{
ORCID iDs \\ Li Yang \\ https://orcid.org/0000-0001-8373-8198 \\ Tao Li \\ https://orcid.org/0000-0001-6689-2951 \\ Tian Tang \\ https://orcid.org/0000-0002-3094-5211 \\ Xinghua Zhang \\ https://orcid.org/0000-0002-1482-1235 \\ Xueping Li \\ https://orcid.org/0000-0002-9406-1861 \\ Chuncai Luo \\ https://orcid.org/0000-0001-5602-6320
}

\section{REFERENCES}

1. Smith-Bindman R. Is computed tomography safe? N Engl J Med 2010;363:1-4

2. Leipsic J, Heilbron BG, Hague C. Iterative reconstruction for coronary CT angiography: finding its way. Int J CardiovasC Imaging 2012;28:613-620

3. Silva AC, Lawder HJ, Hara A, Kujak J, Pavlicek W. Innovations in $\mathrm{CT}$ dose reduction strategy: application of the adaptive statistical iterative reconstruction algorithm. AJR Am J Roentgenol 2010;194:191-199
4. Hou Y, Liu X, Xv S, Guo W, Guo Q. Comparisons of image quality and radiation dose between iterative reconstruction and filtered back projection reconstruction algorithms in 256-MDCT coronary angiography. AJR Am J Roentgenol 2012;199:588-594

5. Klink T, Obmann V, Heverhagen J, Stork A, Adam G, Begemann $P$. Reducing CT radiation dose with iterative reconstruction algorithms: the influence of scan and reconstruction parameters on image quality and CTDIvol. Eur J Radiol 2014;83:1645-1654

6. Leipsic J, Labounty TM, Heilbron B, Min JK, Mancini GB, Lin FY, et al. Adaptive statistical iterative reconstruction: assessment of image noise and image quality in coronary CT angiography. AJR Am J Roentgenol 2010;195:649-654

7. Kröpil P, Bigdeli AH, Nagel HD, Antoch G, Cohnen M. Impact of increasing levels of advanced iterative reconstruction on image quality in low-dose cardiac CT angiography. Rofo 2014;186:567-575

8. Wang R, Schoepf UJ, Wu R, Reddy RP, Zhang C, Yu W, et al. Image quality and radiation dose of low dose coronary CT angiography in obese patients: sinogram affirmed iterative reconstruction versus filtered back projection. Eur J Radiol 2012;81:3141-3145

9. Yuki $H$, Utsunomiya $D$, Funama $Y$, Tokuyasu $S$, Namimoto T, Hirai T, et al. Value of knowledge-based iterative model reconstruction in low-kV 256-slice coronary CT angiography. $J$ Cardiovasc Comput Tomogr 2014;8:115-123

10. Halpern EJ, Gingold EL, White H, Read K. Evaluation of coronary artery image quality with knowledge-based iterative model reconstruction. Acad Radiol 2014;21:805-811

11. Oda S, Weissman G, Vembar M, Weigold WG. Iterative model reconstruction: improved image quality of low-tube-voltage prospective ECG-gated coronary CT angiography images at 256-slice CT. Eur J Radiol 2014;83:1408-1415

12. Oda S, Utsunomiya D, Funama Y, Katahira K, Honda K, Tokuyasu S, et al. A knowledge-based iterative model reconstruction algorithm: can super-low-dose cardiac CT be applicable in clinical settings? Acad Radiol 2014;21:104-110

13. Zhang F, Yang L, Song X, Li YN, Jiang Y, Zhang XH, et al. Feasibility study of low tube voltage $(80 \mathrm{kVp})$ coronary CT angiography combined with contrast medium reduction using iterative model reconstruction (IMR) on standard BMI patients. Br J Radiol 2016;89:20150766

14. Lee J, Park CH, Oh CS, Han K, Kim TH. Coronary computed tomographic angiography at $80 \mathrm{kVp}$ and knowledge-based iterative model reconstruction is non-inferior to that at 100 kVp with iterative reconstruction. PLoS One 2016;11:e0163410

15. Virmani R, Burke AP, Farb A, Kolodgie FD. Pathology of the vulnerable plaque. J Am Coll Cardiol 2006;47(8 Suppl):C13-C18

16. Leipsic J, Abbara S, Achenbach S, Cury R, Earls JP, Mancini GJ, et al. SCCT guidelines for the interpretation and reporting of coronary CT angiography: a report of the Society of Cardiovascular Computed Tomography Guidelines Committee. J Cardiovasc Comput Tomogr 2014;8:342-358 
17. Feuchtner G, Kerber J, Burghard P, Dichtl W, Friedrich G, Bonaros $\mathrm{N}$, et al. The high-risk criteria low-attenuation plaque $<60 \mathrm{HU}$ and the napkin-ring sign are the most powerful predictors of MACE: a long-term follow-up study. Eur Heart J Cardiovasc Imaging 2017;18:772-779

18. Schmid M, Pflederer T, Jang IK, Ropers D, Sei K, Daniel WG, et al. Relationship between degree of remodeling and CT attenuation of plaque in coronary atherosclerotic lesions: an in-vivo analysis by multi-detector computed tomography. Atherosclerosis 2008;197:457-464

19. Hu MQ, Li M, Liu ZY, Huang MP, Liu H, Liang CH. Image quality evaluation of iterative model reconstruction on low tube voltage ( $80 \mathrm{kVp}$ ) coronary CT angiography in an animal study. Acta Radiol 2016;57:170-177

20. Chang W, Lee JM, Lee K, Yoon JH, Yu MH, Han JK, et al. Assessment of a model-based, iterative reconstruction algorithm (MBIR) regarding image quality and dose reduction in liver computed tomography. Invest Radiol 2013;48:598-606

21. Hausleiter J, Martinoff S, Hadamitzky M, Martuscelli E, Pschierer I, Feuchtner GM, et al. Image quality and radiation exposure with a low tube voltage protocol for coronary
CT angiography results of the PROTECTION II Trial. JACC

Cardiovasc Imaging 2010;3:1113-1123

22. Feuchtner GM, Jodocy D, Klauser A, Haberfellner B, Aglan I, Spoeck $A$, et al. Radiation dose reduction by using 100kV tube voltage in cardiac 64-slice computed tomography: a comparative study. Eur J Radiol 2010;75:e51-e56

23. Park CH, Lee J, Oh C, Han KH, Kim TH. The feasibility of submillisievert coronary CT angiography with low tube voltage, prospective ECG gating, and a knowledge-based iterative model reconstruction algorithm. Int J Cardiovasc Imaging 2015;31 Suppl 2:197-203

24. André F, Fortner P, Vembar M, Mueller D, Stiller W, Buss SJ, et al. Improved image quality with simultaneously reduced radiation exposure: knowledge-based iterative model reconstruction algorithms for coronary CT angiography in a clinical setting. J Cardiovasc Comput Tomogr 2017;11:213-220

25. Iyama Y, Nakaura T, Yokoyama K, Kidoh M, Harada K, Oda S, et al. Low-contrast and low-radiation dose protocol in cardiac computed tomography: usefulness of low tube voltage and knowledge-based iterative model reconstruction algorithm. J Comput Assist Tomogr 2016;40:941-947 